\title{
Investigating the efficiency limitations of GaN-based emitters
}

Benjamin. G. Crutchley", Igor P. Marko, Alf R. Adams and Stephen J. Sweeney Advanced Technology Institute and Department of Physics, University of Surrey, Guildford, Surrey, GU27XH, U.K.

*E-mail:b.crutchley@surrey.ac.uk

In this study low temperature and high pressure techniques have been used to investigate the recombination processes taking place in InGaN-based quantum well light emitting diodes (LEDs) which have emission across the blue-green region. Despite relatively high peak efficiencies of the GaN-based emitters, there remain issues relating to the strong efficiency reduction at higher currents that are required for normal operation in most applications. It is observed that there is a relative reduction in efficiency as injection current is increased in a phenonmenon which is known as efficiency droop. There are three main arguments for the cause of efficiency droop that are discussed in the literature: non-radiative Auger recombination, carrier leakage and a defect-related loss mechanism. In spite of extensive research to date, there is little agreement on the cause of efficiency droop as most experiments can only measure the overall efficiency behaviour leading to difficulties in determining the individual contributions from the different loss mechanisms.

Temperature-dependent electroluminescence measurements result in changes to the carrier transport within the LEDs and may therefore be used to understand the impact of carrier injection on efficiency droop in InGaN. High hydrostatic pressure is also applied to the devices in order to identify the dominant loss mechanism as each of the candidate droop-causing mechanisms has a characteristic pressure dependence. The application of high pressure was found to cause a reduction in efficiency over the entire current range but has little effect on efficiency droop (Figures 1 and 2). Such behavior is unlikely to be explained by using an Auger recombination or carrier leakage argument, but is consistent with a carrier-density dependent defect-related loss mechanism causing the onset of efficiency droop. The onset of efficiency droop consequently occurs at a low current where carriers occupy higher energy regions of increased defect density.

An unbiased analysis will be discussed at the conference to determine the relative importance of the candidate loss mechanisms which may be responsible for the efficiency droop that occurs in InGaN-based LEDs.

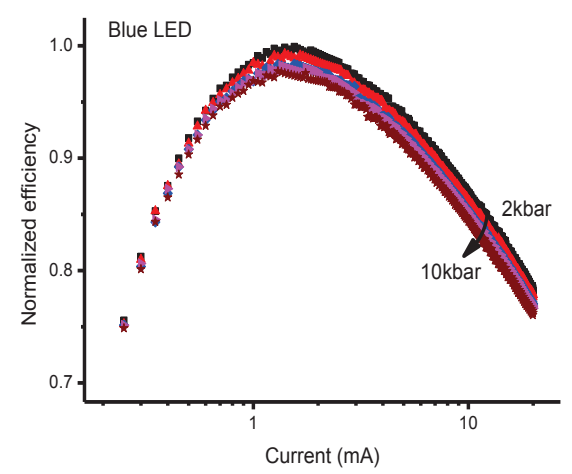

Figure 1- The pressure dependence of efficiency droop for a blue LED.

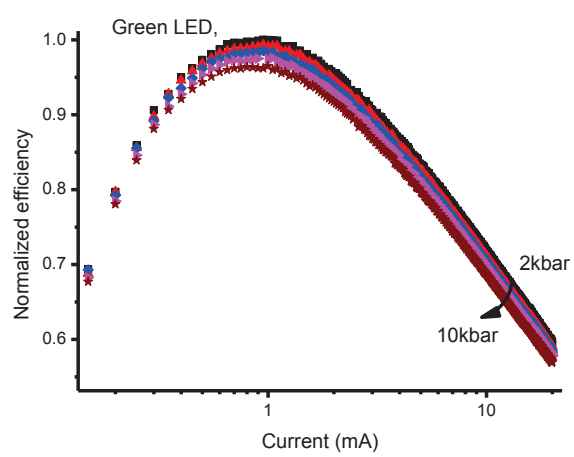

Figure 2- The pressure dependence of efficiency droop for a green LED. 